\title{
Well-posedness for nonlinear Schrödinger equations with boundary forces in low dimensions by Strichartz estimates
}

\author{
Türker Özsarı \\ Department of Mathematics, Izmir Institute of Technology, Izmir 35430, Turkey
}

\section{A R T I C L E I N F O}

\section{Article history:}

Received 29 September 2013

Available online 18 November 2014

Submitted by B. Kaltenbacher

\section{Keywords:}

Nonlinear Schrödinger equations

Inhomogeneous boundary conditions

Strichartz estimates

Local and global existence

Conditional uniqueness

Local stability

\begin{abstract}
A B S T R A C T
In this paper, we study the well-posedness of solutions for nonlinear Schrödinger equations on one and two dimensional domains with boundary where the boundary is disturbed by an external inhomogeneous type of Dirichlet or Neumann force. We first prove the local existence of solutions at the energy level for quadratic and superquadratic sources using the Strichartz estimates on domains. Secondly, we obtain conditional uniqueness and local stability. Then, we prove the boundedness of solutions in the energy space to pass from the local theory to the global theory. Regarding subquadratic sources, we appeal to classical methods and Trudinger's inequality to prove the uniqueness, which, combined with the existence of weak energy solutions, mass and energy inequalities, eventually implies the continuity of solutions in time.
\end{abstract}

() 2014 Elsevier Inc. All rights reserved.

\section{Introduction}

\subsection{Problem description and motivation}

In this paper, we consider the following nonlinear Schrödinger equation (NLS) with an inhomogeneous Dirichlet or Neumann boundary condition:

$$
\begin{cases}D_{t} u+\Delta u+\lambda|u|^{r} u=0 & \text { in } Q=(0, T) \times M ; \\ u(0)=u_{0} & \text { in } M ; \\ B u=h & \text { in } \Sigma=(0, T) \times \partial M .\end{cases}
$$

In (1), $M$ is a general bounded domain in $\mathbb{R}^{d}$ with smooth boundary $\Gamma \equiv \partial M$. We assume $d \leq 2$, except in Theorem 1.3 below, which is true for any $d \geq 1$. In the special case where $M$ is a Riemaniann manifold, one replaces the Laplacian with the Laplace-Beltrami operator $\Delta_{g}$, where $g$ is the metric on $M$. In (1),

E-mail address: turkerozsari@iyte.edu.tr.

URL: http://web.iyte.edu.tr/ turkerozsari/. 
$\lambda \in\{-1,1\}, r>0, D_{t}=i^{-1} \partial_{t}$, and $u=u(t, x)$ is a complex valued function. $B$ is either a Dirichlet or Neumann boundary operator, i.e., $B u=u$ (Dirichlet) or $B u=\frac{\partial u}{\partial n}$ (Neumann), where $\frac{\partial}{\partial n}$ denotes the outward unit normal derivative. (1) is said to be focusing (resp. defocusing) if $\lambda=1$ (resp. $\lambda=-1$ ).

The existence of a weak solution in the space $L^{\infty}\left(0, T ; H^{1}(M)\right)$ for (1) with a Dirichlet boundary condition has been proved in [19] (with $\lambda=-1, r>0$ ) and in [4] (with $\lambda=1, r \leq \frac{2}{d}$ ). In both works, the authors assume that $d \geq 1$ and $h \in C^{3}(\Sigma)$ together with the compatibility condition $\left.u_{0}\right|_{\partial M}=h(0)$. These results have been extended to NLS with linear damping (iau, $a>0)$ and rougher boundary data $\left(h \in H^{1}(\Sigma) \cap\right.$ $\left.L^{r+2}(\Sigma)\right)$ for $\lambda=-1, r>0$ in [16] and for $\lambda=1, r<\frac{4}{d+2}$ in [17]. NLS with Neumann type boundary data $\left(h \in C^{1}\left([0, T] ; L^{2}(\Gamma)\right)\right)$ and linear damping $(i a u, a>0)$ has recently been treated in [18] in both the focusing case $\left(\lambda=1, r<\frac{4}{d+2}\right)$ and the defocusing case $(\lambda=-1, r>0)$. Ref. [18] also studies NLS with nonlinear damping $i a|u|^{s} u, a>0,0<r<s<1$ with boundary force of type $h \in C^{1}\left([0, T] ; L^{\frac{2}{1-s}}(\Gamma)\right)$. Moreover, Refs. [16-18] obtain the stabilization of solutions with respect to the behavior of the boundary force.

To the best of our knowledge, there is not yet a well established well-posedness theory for NLS with boundary forces on bounded domains at the $H^{1}$-level in low dimensions. There are some results on special unbounded domains with boundary such as the half-line; for instance Refs. [8-11,20] study one-dimensional NLS with boundary forces on such a domain. There are also some results on related equations such as Ginzburg-Landau equations with boundary forces; see for example $[2,6,7,15]$. In high dimensions $(d \geq 3)$, well-posedness for NLS on bounded domains, even with homogeneous boundary datum $h \equiv 0$, has not been studied well yet.

It has been pointed out in [19] that the uniqueness and the regularity for (1) are non-trivial open problems. However, it is stated in [19] that if $r<4 /(d-2)$ and the evolution operator satisfies the decay property

$$
\left\|e^{i t \Delta}\right\|_{\mathcal{L}\left(L^{1}(M), L^{\infty}(M)\right)} \lesssim t^{-d / 2},
$$

then there must be a unique solution. Although such a decay property is not known to hold on a general domain, similar estimates, so called Strichartz estimates, have been proved recently on manifolds with boundary [3] and low dimensional compact regular domains [1].

It is proved in [3] that for any Strichartz pair $(p, q)$, the Strichartz estimate

$$
\left\|e^{i t \Delta_{g}} f\right\|_{L^{p}\left(0, T ; L^{q}(M)\right)} \lesssim\|f\|_{H^{\frac{4}{3 p}}(M)}
$$

holds true in a compact manifold with boundary with dimension $d \geq 2$.

We say $(p, q)$ is a Strichartz pair if

$$
\frac{2}{p}+\frac{d}{q}=\frac{d}{2}, \quad(p, q, d) \neq(2, \infty, 2), 2 \leq p, q \leq \infty
$$

Regarding a general domain, Ref. [1] proved the following Strichartz estimate where $M$ is a two or three dimensional compact regular domain.

$$
\left\|e^{i t \Delta} f\right\|_{L^{p}\left(0, T ; L^{q}(M)\right)} \lesssim\|f\|_{H^{\frac{3}{2 p}+\epsilon}(M)},
$$

$\epsilon>0$ being an arbitrarily small positive constant. Observe that there is a loss of $\frac{1}{6 p}+\epsilon$ derivatives in the estimate (3) compared to the estimate (2).

In two dimensional domains, using Sobolev imbeddings it is possible to improve (3) to the case $q=\infty$ [1], in which case we have

$$
\left\|e^{i t \Delta} f\right\|_{L^{p}\left(0, T ; L^{\infty}(M)\right)} \lesssim\|f\|_{H^{1}(M)} .
$$

The above Strichartz estimate is valid for both Neumann and Dirichlet boundary conditions. 


\subsection{Main results}

For the Dirichlet problem and $d=2$, we consider the following class of boundary functions:

$$
\begin{aligned}
\mathbf{D} \equiv & \left\{h \in H^{1+\epsilon}(\Sigma) \cap L^{r+2}(\Sigma):|h|^{r} h \in L^{1}\left(0, T ; H^{\frac{1}{2}+\epsilon}(\Gamma)\right),\right. \\
& \left.D_{t}^{\epsilon}\left(|h|^{r} h\right) \in L^{1}\left(0, T ; H^{\frac{1}{2}+\epsilon}(\Gamma)\right), h(0) \in H^{\frac{1}{2}+\epsilon}(\Gamma)\right\},
\end{aligned}
$$

where $\epsilon$ is an arbitrarily small fixed positive real number, $D_{t}^{\epsilon}$ is the fractional time derivative, i.e., $|h|^{r} h \in$ $W^{\epsilon, 1}\left(0, T ; H^{\frac{1}{2}+\epsilon}(\Gamma)\right)$ and $H^{1+\epsilon}(\Sigma) \equiv H^{1+\epsilon}\left(0, T ; L^{2}(\Gamma)\right) \cap L^{2}\left(0, T ; H^{1+\epsilon}(\Gamma)\right)$. The space $\mathbf{D}$ can be written in a more compact form. Especially in low dimensions after some elimination by using the interpolation theory and Sobolev imbeddings on a manifold, we omit this discussion.

For the Neumann problem and $d=2$, we consider the following class of boundary functions

$$
\mathbf{N} \equiv C^{1}\left([0, T] ; L_{2}(\Gamma)\right)
$$

For $d=1$, we assume $M=(0,1)$. In this case, the boundary is composed of two end points $\Gamma=\{0,1\}$. Therefore, the boundary condition can be split into two functions, namely

$$
h(t, x)= \begin{cases}h_{0}(t), & \text { if } x=0 \\ h_{1}(t), & \text { if } x=1\end{cases}
$$

For $d=2$ we set $X_{T} \equiv C\left([0, T] ; H^{1}(M)\right) \cap L^{p}\left(0, T ; L^{\infty}(M)\right)$ where $p>\max \{2, r+1\}$. We equip $X_{T}$ with the norm

$$
\|u\|_{X_{T}} \equiv \sup _{t \in[0, T]}\left(\|u(t)\|_{H^{1}(M)}+\|u\|_{L^{p}\left(0, T ; L^{\infty}(\Omega)\right)}\right) .
$$

For $d=1$, we set $X_{T} \equiv C\left([0, T] ; H^{1}(M)\right)$ and equip it with the norm

$$
\|u\|_{X_{T}} \equiv \sup _{t \in[0, T]}\|u(t)\|_{H^{1}(M)} .
$$

In both cases, $X_{T}$ is a Banach space.

Sometimes, we will write $X_{T}^{d}$ for $X_{T}$ to emphasize the dimension. Here are our main results.

Theorem 1.1 (Global existence, conditional uniqueness). Let $u_{0} \in H^{1}(M), r>0$ if $\lambda=-1$ (defocusing), $0<r<\frac{4}{d+2}$ if $\lambda=1$ (focusing), and one of the following conditions holds

(i) $B=i d$ (Dirichlet problem), $d=2, h \in \mathbf{D},\left.u_{0}\right|_{\partial M} \equiv h(0)$,

(ii) $B=\frac{\partial}{\partial n}$ (Neumann problem), $d=2, h \in \mathbf{N}$,

(iii) $B=i d$ (Dirichlet problem), $d=1, h_{i} \in H^{1}(0, T), i=0,1,\left.u_{0}\right|_{\partial M} \equiv h(0)$,

(iv) $B=\frac{\partial}{\partial n}$ (Neumann problem), $d=1, h_{i} \in C^{1}([0, T]), i=0,1$,

then (1) has a unique solution $u \in X_{T}^{d}$.

Remark 1. (i) Regarding the above theorem, the local existence of a strong $H^{1}$-solution for $r \geq 1$ is proved in Section 3.1.1 and Section 3.1.2. The uniqueness of this local solution is proved in Lemma 3.4. The uniform boundedness of the $H^{1}$-norm is proved in Section 3.3 in order to extend this local solution globally. For $r<1$ (even for $r \leq 2$ ), we prove the uniqueness of a global weak $H^{1}$-solution in Section 3.5. Then by Theorem 1.3, the existence of a strong $H^{1}$-solution follows. 
(ii) We say the uniqueness is conditional if it holds only in a strict subspace of the function space $C\left([0, T] ; H^{1}(M)\right)$. The uniqueness result in the above theorem is conditional only for $r>2$ and $d=2$. In other cases such as $d=1$ or $r \leq 2, d=2$, uniqueness holds in the entire space $C\left([0, T] ; H^{1}(M)\right)$.

Theorem 1.2 (Local stability). Let $d \leq 2, r>1$ and $O \ni\left(u_{0}, h\right)$ be a bounded set associated with the spaces given in Theorem 1.1(i)-(iv) for the Dirichlet or Neumann problem. Then, there exists $T>0$ such that the flow $\left(u_{0}, h\right) \rightarrow u$ is Lipschitz from $O$ into $C\left([0, T] ; H^{1}(M)\right)$.

\section{Remark 2.}

(i) The proof of Theorem 1.2 is given in Section 3.2.

(ii) In order to give a sense to the boundedness of a subset of $\mathbf{D}$, one can define a metric on $\mathbf{D}$ using a norm like function $\|\cdot\|_{\mathbf{D}}: \mathbf{D} \rightarrow[0, \infty)$ such as

$$
\|h\|_{\mathbf{D}} \equiv\|h\|_{H^{1+\epsilon}(\Sigma) \cap L^{r+2}(\Sigma)}+\left\||h|^{r} h\right\|_{L^{1}\left(0, T ; H^{\frac{1}{2}+\epsilon}(\Gamma)\right)}+\left\||h|^{r} h\right\|_{W^{\epsilon, 1}\left(0, T ; H^{\frac{1}{2}+\epsilon}(\Gamma)\right)}+\|h(0)\|_{H^{\frac{1}{2}+\epsilon}(\Gamma)} .
$$

Our proof method for Theorem 1.1 is based on Strichartz estimates, which work well for $r \geq 1$. Regarding the case $r<1$ (or even $r \leq 2$ ), we first prove that if (1) has a unique solution which is essentially bounded in time, then it must be continuous in time. This argument is true for any dimension $d \geq 1$. More precisely, we have the following theorem.

Theorem 1.3 (Continuity in time). Let $d \geq 1$ and $u$ be a unique weak $H^{1}$-solution of $(1)$ in $L^{\infty}\left(0, T ; H^{1}(M)\right) \cap$ $W^{1, \infty}\left(0, T ;\left[H^{1}(M)\right]^{\prime}\right)$. Then, $u$ is a unique strong $H^{1}$-solution of $(1)$ in $C\left([0, T] ; H^{1}(M)\right) \cap C^{1}(0, T$; $\left.\left[H^{1}(M)\right]^{\prime}\right)$.

\section{Remark 3.}

(i) Theorem 1.3 is proved in Section 3.4.

(ii) See Definition 3.1 for the precise notion of weak and strong $H^{1}$-solutions for (1).

An intuitive approach for solving partial differential equations with boundary forces is homogenization by extending the boundary datum. For example, in [19] the authors extend the very regular $\left(C^{3}\right)$ boundary datum $h$ to the interior as some smooth $\tilde{h}$ which satisfies several trace conditions involving derivatives on the boundary, and then subtract this extension from the main equation. We consider physically more reasonable (rougher) boundary data as opposed to the very regular $\left(C^{3}\right)$ boundary inputs considered in the previous literature $[15,19]$. This is one of the distinctive features of our paper because one cannot always find a very nice extension when the boundary input is taken from a rougher function space. For example, according to the classical trace theory one only gains $\frac{1}{2}$ derivatives by Dirichlet extension. This is not sufficient to define traces such as $\left.\Delta \tilde{h}\right|_{\Gamma}$ used in [19] if $h$ is rough. Therefore, the homogenization method of [19] does not work well for our model. In order to deal with this issue, we will appeal to the dynamic extension method introduced in $[16,17]$. This is simply extending the boundary input as a solution of an associated linear problem which we can solve with rougher boundary conditions. In other words, we are extending the boundary datum in a weak sense. The linear theory required for this extension approach will be reviewed in Section 2.

Secondly, we prove the local existence and uniqueness of a strong $H^{1}$-solution for both Dirichlet and Neumann problems with both focusing and defocusing power type nonlinearities by using a contraction mapping argument which uses the Strichartz estimate (4) in $d=2$. This fixed point approach works for powers $r \geq 1$. Regarding powers $r>2$ and $d=2$, uniqueness is only shown to hold in a conditional sense, 
namely in the space $X_{T}$. In order to extend the local solution globally in time, we prove the boundedness of the solutions in $H^{1}$-sense. For the Dirichlet problem, this is done by controlling the trace of the directional derivative of the solutions. We can manage to bound solutions in $H^{1}$-sense for $r>0$ in the defocusing case. However, the focusing problem is more difficult due to the fact that energy identities involve nonlinear terms with a bad sign, which might eventually cause the blow-up of the solutions, even in the case of homogeneous boundary conditions [12]. One can utilize Gagliardo-Nirenberg and Gronwall type estimates to overcome this difficulty for nonlinearities with powers up to $r<\frac{4}{d+2}$. Unfortunately, this corresponds to $r<\frac{4}{3}$ for $d=1$ and $r<1$ for $d=2$, which are not completely included in the fixed point approach. Fortunately, we know that (1) has weak $H^{1}$-solutions in this case $[17,18]$. Moreover, one can prove that there is indeed only one weak $H^{1}$-solution by using Sobolev imbedding $H^{1} \hookrightarrow L^{\infty}$ in $d=1$ and Trudinger's inequality in $d=2$. Uniqueness combined with mass and energy inequalities gives the regularity and implies that the unique weak $H^{1}$-solution is indeed a strong $H^{1}$-solution.

Remark 4. In the case of homogeneous boundary conditions and arbitrarily large initial datum, weak solutions globally exist for powers $r<\frac{4}{d}$. Therefore, the gap $r \in\left[\frac{4}{d+2}, \frac{4}{d}\right)$ remains an interesting open problem for the focusing NLS with non-homogeneous boundary conditions.

\section{Review of linear theory}

In this section, we review main regularity results for the linear dynamics.

\subsection{Dirichlet problem}

We consider the following linear Schrödinger equation with an inhomogeneous Dirichlet boundary condition:

$$
\begin{cases}D_{t} v+\Delta v+z=0 & \text { in } Q=(0, T) \times M \\ v(0)=v_{0} & \text { in } M ; \\ u=h & \text { in } \Sigma=(0, T) \times \partial M\end{cases}
$$

In the Dirichlet problem, we know that there exists a unique solution $v \in C\left([0, T] ; H^{1}(M)\right)$ provided that $z \in L^{1}\left(0, T ; H^{1}(M)\right), v_{0} \in H^{1}(M)$, and $h \in H^{1}(\Sigma)$ with the compatibility relation

$$
\left.v_{0}\right|_{\partial M}=h(0) \in H^{\frac{1}{2}}(\partial M) .
$$

Moreover, solutions depend continuously on data. That is, the mapping

$$
\left(v_{0}, h, z\right) \rightarrow v
$$

is (Lipschitz) continuous from the subspace of elements of

$$
H^{1}(M) \times H^{1}(\Sigma) \times L^{1}\left(0, T ; H^{1}(M)\right)
$$

satisfying (6) into $C\left([0, T] ; H^{1}(M)\right)$. For details, see [14, Section 10.9]. We will need a little bit more regularity in $d=2$ in order to use the Sobolev imbedding $H^{1+\epsilon} \hookrightarrow L^{\infty}$. Indeed, it is possible to shift $H^{1}$-regularity up by one unit [17]. Namely, there is a unique solution $v \in C\left([0, T] ; H^{2}(M)\right)$ provided that $z \in L^{1}\left(0, T ; H^{2}(M)\right), z_{t} \in L^{1}\left(0, T ; H^{1}(M)\right), v_{0} \in H^{2}(M), h \in H^{2}(\Sigma)$ with the compatibility relation $\left.v_{0}\right|_{\partial M}=h(0) \in H^{\frac{3}{2}}(\partial M)$. Again the mapping from data space to solution space is continuous. Moreover, one can interpolate between the two cases, above and obtain a unique solution $v \in C\left([0, T] ; H^{1+\epsilon}(M)\right)$ continuously, provided that $z \in L^{1}\left(0, T ; H^{1+\epsilon}(M)\right), D_{t}^{\epsilon} z \in L^{1}\left(0, T ; H^{1}(M)\right), v_{0} \in H^{1+\epsilon}(M), h \in H^{1+\epsilon}(\Sigma)$ with the compatibility relation $\left.v_{0}\right|_{\partial M}=h(0) \in H^{\frac{1}{2}+\epsilon}(\partial M)$. See for example $[13,14]$ for interpolation. 


\subsection{Neumann problem}

We consider the following linear Schrödinger equation with an inhomogeneous Neumann boundary condition:

$$
\begin{cases}D_{t} v+\Delta v=0 & \text { in } Q=(0, T) \times M \\ v(0)=v_{0} & \text { in } M \\ \frac{\partial v}{\partial n}=h & \text { in } \Sigma=(0, T) \times \partial M\end{cases}
$$

In the Neumann problem, we have recently proved in [18] that $v \in C\left([0, T] ; H^{1}(M)\right)$ continuously provided that $h \in C^{1}\left([0, T] ; L_{2}(\Gamma)\right)$ and $v_{0} \in H^{1}(M)$. Actually, this result can be achieved even with $h \in C^{1}\left([0, T] ; H^{-\frac{1}{2}}(\Gamma)\right)$. One can shift this regularity result up and get $v \in C\left([0, T] ; H^{3}(M)\right)$ continuously by choosing $h \in C^{1}\left([0, T] ; H^{\frac{3}{2}}(\Gamma)\right), v_{0} \in H^{3}(M)$. Then by interpolation one gets $v \in C\left([0, T] ; H^{1+\epsilon}(M)\right)$ continuously provided that $h \in C^{1}\left([0, T] ; H^{-\frac{1}{2}+\epsilon}(\Gamma)\right), v_{0} \in H^{1+\epsilon}(M)$. This boundary regularity is a fortiori satisfied by our assumption of $h \in C^{1}\left([0, T] ; L^{2}(\Gamma)\right)=\mathbf{N}$.

\section{Nonlinear theory}

\subsection{Quadratic and superquadratic sources}

Let $r \geq 1$ and suppose one of the conditions (i) to (iv) in Theorem 1.1 is satisfied. Let us define $v$ as the unique solution of the linear dynamics (5) or (7) for the Dirichlet or the Neumann problem, respectively. For $d=2$, the initial datum $v_{0}$ in (5) and (7) is chosen from the space $H^{1+\epsilon}(M)$. For the Dirichlet problem, it is chosen in such a way that $\left.v_{0}\right|_{\Gamma}=h(0)$. Similarly, for the Neumann problem we can choose $v_{0}$ in such a way that $\frac{\partial v_{0}}{\partial n}=h(0)$. Existence of such $v_{0}$ follows by the Sobolev trace theory. In the case $d=1$, there is no harm to take $v_{0}=u_{0} \in H^{1}(M)$.

Now, (1) is reduced to solving

$$
\begin{cases}D_{t} w+\Delta w+f_{v, z}(w)=0 & \text { in } Q=(0, T) \times M \\ w(0)=u_{0}-v_{0} & \text { in } M ; \\ B w=0 & \text { in } \Sigma=(0, T) \times \partial M\end{cases}
$$

where $f_{v, z}(w) \equiv \lambda|w+v|^{r}(w+v)-z$ where we take $z \equiv \gamma_{0}^{-}\left(|h|^{r} h\right)$ for the Dirichlet problem and $z \equiv 0$ for the Neumann problem. Here, $\gamma_{0}^{-}$is the bounded right inverse of the trace operator. This particular choice of $z$ makes $f$ vanish on the boundary for the Dirichlet problem. This is needed in order to utilize Strichartz estimates.

Throughout the paper, we adapt to the following weak and strong notions of $H^{1}$-solutions.

Definition 3.1. $u=v+w$ is said to be a weak $H^{1}$-solution of the Dirichlet (resp. Neumann) problem (1) on $[0, T)$ if $w \in L^{\infty}\left(0, T ; H_{0}^{1}(M)\right) \cap W^{1, \infty}\left(0, T ; H^{-1}(M)\right)\left(\right.$ resp. $\left.w \in L^{\infty}\left(0, T ; H^{1}(M)\right) \cap W^{1, \infty}\left(0, T ; H^{1}(M)^{\prime}\right)\right)$, $D_{t} w+\Delta w+f_{v, z}(w)=0$ in $H^{-1}(M)$ (resp. $\left.H^{1}(M)^{\prime}\right)$ for a.a. $t \in[0, T), w(0)=u_{0}-v_{0}$.

Similarly, $u=v+w$ is said to be a strong $H^{1}$-solution of the Dirichlet (resp. Neumann) problem (1) on $[0, T)$ if $w \in C\left([0, T) ; H_{0}^{1}(M)\right) \cap C^{1}\left((0, T) ; H^{-1}(M)\right)\left(\operatorname{resp} . C\left([0, T) ; H^{1}(M)\right) \cap C^{1}\left((0, T) ; H^{1}(M)^{\prime}\right)\right)$, $D_{t} w+\Delta w+f_{v, z}(w)=0$ in $H^{-1}(M)\left(\operatorname{resp} . H^{1}(M)^{\prime}\right)$ for all $t \in[0, T), w(0)=u_{0}-v_{0}$. 
Remark 5. By Duhamel's formula, it follows that $u$ is a weak (resp. strong) $H^{1}$-solution of $(1)$ on $[0, T)$ if $u \in L^{\infty}\left(0, T ; H^{1}(M)\right)$ (resp. $\left.u \in C\left([0, T) ; H^{1}(M)\right)\right)$ and

$$
u(t)=v(t)+e^{-i t \Delta}\left(u_{0}-v_{0}\right)-i \int_{0}^{t} e^{-i(t-s) \Delta} f_{z}(u) d s \text { for a.a. (resp. for all) } t \in[0, T) .
$$

In order to obtain local solutions we will use the fixed point method. Now, we define the mapping $\Psi$ by

$$
[\Psi(u)](t) \equiv v(t)+e^{-i t \Delta}\left(u_{0}-v_{0}\right)-i \int_{0}^{t} e^{-i(t-s) \Delta} f_{z}(u) d s
$$

where $f_{z}(u)=|u|^{r} u-z=f_{z, v}(w)$. We want to show that $\Psi$ is a contraction on a complete subspace of $X_{T}$ for sufficiently small $T$, from which it will follow that it has a fixed point, i.e., a local solution. In order to achieve this, we will need Lemma 3.2 and Lemma 3.3 below.

Lemma 3.2. Let $u \in X_{T}$ and $p>r+1$. Then,

(i) $\int_{0}^{T}\left\||u|^{r} u\right\|_{H^{1}(M)} d t \leq C T\|u\|_{X_{T}}^{r+1}$ if $d=1$.

(ii) $\int_{0}^{T}\left\||u|^{r} u\right\|_{H^{1}(M)} d t \leq\left(T^{\frac{p-r-1}{p}}+(r+1) T^{\frac{p-r}{p}}\right)\|u\|_{X_{T}}^{r+1}$ if $d=2$.

Proof. Let us first observe that for a.e. $t \geq 0$, we have

$$
\begin{aligned}
\left\||u|^{r} u\right\|_{H^{1}(M)} & =\|u\|_{L^{2 r+2}(M)}^{r+1}+\left\|\frac{r+2}{2}|u|^{r} \nabla u+\frac{r}{2}|u|^{r-2} u^{2} \nabla \bar{u}\right\|_{L_{2}(M)} \\
& \leq\|u\|_{L^{\infty}(M)}^{r+1}+(r+1)\|u\|_{L^{\infty}(M)}^{r}\|\nabla u\|_{L^{2}(M)} .
\end{aligned}
$$

If we integrate $(10)$ over $[0, T]$, we get

$$
\begin{aligned}
\int_{0}^{T}\left\||u|^{r} u\right\|_{H^{1}(M)} d t & \leq \int_{0}^{T}\left(\|u\|_{L^{\infty}(M)}^{r+1}+(r+1)\|u\|_{L^{\infty}(M)}^{r}\|\nabla u\|_{L^{2}(M)}\right) d t \\
& =\int_{0}^{T}\|u\|_{L^{\infty}(M)}^{r+1} d t+(r+1) \int_{0}^{T}\|u\|_{L^{\infty}(M)}^{r}\|\nabla u\|_{L^{2}(M)} d t \leq C T\|u\|_{X_{T}}^{r+1} .
\end{aligned}
$$

In $d=1$, we use the Sobolev imbedding $H^{1} \hookrightarrow L^{\infty}$ to bound the right hand side of (11) by $C T\|u\|_{X_{T}}^{r+1}$. This proves (i).

In $d=2$, we use Hölder's inequality to get

$$
\int_{0}^{T}\|u\|_{L^{\infty}(M)}^{r+1} d t \leq\left(\int_{0}^{T}\|u\|_{L^{\infty}(M)}^{p} d t\right)^{\frac{r+1}{p}}\left(\int_{0}^{T} d t\right)^{\frac{p-r-1}{p}} \leq T^{\frac{p-r-1}{p}}\|u\|_{X_{T}}^{r+1}
$$

and

$$
\begin{aligned}
\int_{0}^{T}\|u\|_{L^{\infty}(M)}^{r}\|\nabla u\|_{L^{2}(M)} d t & \leq\|u\|_{C\left([0, T] ; H^{1}(M)\right)}\left(\int_{0}^{T}\|u\|_{L^{\infty}(M)}^{p} d t\right)^{\frac{r}{p}}\left(\int_{0}^{T} d t\right)^{\frac{p-r}{p}} \\
& \leq T^{\frac{p-r}{p}}\|u\|_{X_{T}}^{r+1} .
\end{aligned}
$$


By (12)-(13), it follows that

$$
\int_{0}^{T}\left\||u|^{r} u\right\|_{H^{1}(M)} d t \leq \overbrace{\left(T^{\frac{p-r-1}{p}}+(r+1) T^{\frac{p-r}{p}}\right)}^{=: C_{r, p, T}}\|u\|_{X_{T}}^{r+1} .
$$

This is (ii).

Lemma 3.3. Let $r \geq 1$ and $u_{1}, u_{2} \in H^{1} \cap L^{\infty}$, then

$$
\begin{aligned}
& \left\|\left|u_{1}\right|^{r} u_{1}-\left|u_{2}\right|^{r} u_{2}\right\|_{H^{1}(M)}^{2} \\
& \quad \leq C\left\|u_{1}-u_{2}\right\|_{H^{1}(M)}^{2}\left(\left\|u_{1}\right\|_{L^{\infty}(M)}+\left\|u_{2}\right\|_{L^{\infty}(M)}\right)^{2 r} \\
& \quad+C\left\|u_{1}-u_{2}\right\|_{L^{\infty}(M)}^{2}\left(\left\|u_{1}\right\|_{L^{\infty}(M)}+\left\|u_{2}\right\|_{L^{\infty}(M)}\right)^{2 r-2}\left(\left\|u_{1}\right\|_{H^{1}(M)}+\left\|u_{2}\right\|_{H^{1}(M)}\right)^{2}
\end{aligned}
$$

Proof. Let's set $g(t) \equiv t u_{1}+(1-t) u_{2}, 0 \leq t \leq 1$. Then

$$
\begin{aligned}
\left\|\left|u_{1}\right|^{r} u_{1}-\left|u_{2}\right|^{r} u_{2}\right\|_{H_{0}^{1}(M)}^{2} & =\int_{M}\left|\nabla\left(\left|u_{1}\right|^{r} u_{1}-\left|u_{2}\right|^{r} u_{2}\right)\right|^{2} d x \\
& =\int_{M}\left|\nabla\left(|g(1)|^{r} g(1)-|g(0)|^{r} g(0)\right)\right|^{2} d x=\int_{M}\left|\nabla \int_{0}^{1} \frac{d}{d t}\left(|g(t)|^{r} g(t)\right) d t\right|^{2} d x \\
& =\int_{M}\left|\nabla\left(\int_{0}^{1}\left(\frac{r+2}{2}|g(t)|^{r} g^{\prime}(t)+\frac{r}{2}|g(t)|^{r-2}(g(t))^{2} \bar{g}^{\prime}(t)\right) d t\right)\right|^{2} d x .
\end{aligned}
$$

We observe the following simple inequalities,

$$
\begin{gathered}
\left.\left.|\nabla| g(t)\right|^{r}|=r| \operatorname{Re}|g(t)|^{r-2} \bar{g}(t) \nabla g(t)|\leq r| g(t)\right|^{r-1}|\nabla g(t)|, \\
|g(t)|^{r}=\left|t u_{1}+(1-t) u_{2}\right|^{r} \leq\left(t\left|u_{1}\right|+(1-t)\left|u_{2}\right|\right)^{r} \leq\left(\left|u_{1}\right|+\left|u_{2}\right|\right)^{r}, \\
|\nabla g(t)|=\left|t \nabla u_{1}+(1-t) \nabla u_{2}\right| \leq\left|\nabla u_{1}\right|+\left|\nabla u_{2}\right|,
\end{gathered}
$$

and

$$
\int_{0}^{1}|g(t)|^{r} d t \leq\left(\left|u_{1}\right|+\left|u_{2}\right|\right)^{r}
$$

Using (17)-(20), we first have

$$
\begin{aligned}
\left|\int_{0}^{1} \nabla\left(|g(t)|^{r} g^{\prime}(t)\right) d t\right| & =\left|\int_{0}^{1}\left(\nabla\left(u_{1}-u_{2}\right)|g(t)|^{r}+\left(u_{1}-u_{2}\right) \nabla|g(t)|^{r}\right) d t\right| \\
& \leq\left|\nabla u_{1}-\nabla u_{2}\right|\left(\left|u_{1}\right|+\left|u_{2}\right|\right)^{r}+r\left|u_{1}-u_{2}\right|\left(\left|u_{1}\right|+\left|u_{2}\right|\right)^{r-1}\left(\left|\nabla u_{1}\right|+\left|\nabla u_{2}\right|\right) .
\end{aligned}
$$

Secondly,

$$
\left|\int_{0}^{1} \nabla\left(|g(t)|^{r-2}(g(t))^{2} \bar{g}^{\prime}(t)\right) d t\right|=\left|\int_{0}^{1}\left(\nabla\left(\bar{u}_{1}-\bar{u}_{2}\right)|g(t)|^{r-2}(g(t))^{2}+\left(\bar{u}_{1}-\bar{u}_{2}\right) \nabla\left(|g(t)|^{r-2}(g(t))^{2}\right)\right) d t\right|
$$




$$
\begin{aligned}
\leq & \left|\nabla u_{1}-\nabla u_{2}\right|\left(\left|u_{1}\right|+\left|u_{2}\right|\right)^{r} \\
& +C\left|u_{1}-u_{2}\right|\left(\left|u_{1}\right|+\left|u_{2}\right|\right)^{r-1}\left(\left|\nabla u_{1}\right|+\left|\nabla u_{2}\right|\right) .
\end{aligned}
$$

Hence,

$$
\begin{aligned}
& \left\|\left|u_{1}\right|^{r} u_{1}-\left|u_{2}\right|^{r} u_{2}\right\|_{H_{0}^{1}(M)}^{2} \\
& \leq C \int_{M}|| \nabla u_{1}-\nabla u_{2}\left|\left(\left|u_{1}\right|+\left|u_{2}\right|\right)^{r}+\right| u_{1}-u_{2}\left|\left(\left|u_{1}\right|+\left|u_{2}\right|\right)^{r-1}\left(\left|\nabla u_{1}\right|+\left|\nabla u_{2}\right|\right)\right|^{2} d x \\
& \leq C \int_{M}\left|\nabla u_{1}-\nabla u_{2}\right|^{2}\left(\left|u_{1}\right|+\left|u_{2}\right|\right)^{2 r} d x+C \int_{M}\left|u_{1}-u_{2}\right|^{2}\left(\left|u_{1}\right|+\left|u_{2}\right|\right)^{2 r-2}\left(\left|\nabla u_{1}\right|+\left|\nabla u_{2}\right|\right)^{2} d x \\
& \leq C\left\|u_{1}-u_{2}\right\|_{H_{0}^{1}(M)}^{2}\left(\left\|u_{1}\right\|_{L^{\infty}(M)}+\left\|u_{2}\right\|_{L^{\infty}(M)}\right)^{2 r} \\
& \quad+C\left\|u_{1}-u_{2}\right\|_{L^{\infty}(M)}^{2}\left(\left\|u_{1}\right\|_{L^{\infty}(M)}+\left\|u_{2}\right\|_{L^{\infty}(M)}\right)^{2 r-2}\left(\left\|u_{1}\right\|_{H^{1}(M)}+\left\|u_{2}\right\|_{H^{1}(M)}\right)^{2} .
\end{aligned}
$$

\subsubsection{One dimensional domains}

Now, given $u \in X_{T}=C\left([0, T] ; H^{1}(M)\right)$, we have

$$
\begin{aligned}
\|\Psi(u)\|_{H^{1}(M)} & =\left\|v(t)-i \int_{0}^{t} e^{-i(t-s) \Delta} f_{z}(u) d s\right\|_{H^{1}(M)} d s \\
& \leq\|v\|_{H^{1}(M)}+\int_{0}^{T}\left\|e^{-i(t-s) \Delta} f_{z}(u)\right\|_{H^{1}(M)} d s \\
& =\|v\|_{H^{1}(M)}+\int_{0}^{T}\left\|f_{z}(u)\right\|_{H^{1}(M)} d s=\|v\|_{H^{1}(M)}+\int_{0}^{T}\left\||u|^{r} u-z\right\|_{H^{1}(M)} d s \\
& \leq\|v\|_{H^{1}(M)}+\int_{0}^{T}\left\||u|^{r} u\right\|_{H^{1}(M)} d s+\int_{0}^{T}\|z\|_{H^{1}(M)} d s \\
& \leq\|v\|_{C\left([0, T] ; H^{1}(M)\right)}+C T\|u\|_{X_{T}}^{r+1}+\|z\|_{L^{1}\left(0, T ; H_{1}(M)\right)} .
\end{aligned}
$$

In the above estimates, we have used the conservation of the Schrödinger flow, the Sobolev imbedding $H^{1}(M) \hookrightarrow L^{\infty}(M)$ in one-dimensional spaces, and Lemma 3.2. By the estimate (24), it follows that $\Psi$ maps $X_{T}$ into $X_{T}$.

Now, given $u_{1}, u_{2} \in X_{T}$, by Lemma 3.3 , we have

$$
\begin{aligned}
\left\|\Psi\left(u_{1}\right)-\Psi\left(u_{2}\right)\right\|_{H_{1}(M)} & =\left\|\int_{0}^{t} e^{-i(t-s) \Delta}\left(\left|u_{1}\right|^{r} u_{1}-\left|u_{2}\right|^{r} u_{2}\right) d s\right\|_{H^{1}(M)} \\
& \leq \int_{0}^{T}\left\|e^{-i(t-s) \Delta}\left(\left|u_{1}\right|^{r} u_{1}-\left|u_{2}\right|^{r} u_{2}\right)\right\|_{H^{1}(M)} d s=\int_{0}^{T}\left\|\left|u_{1}\right|^{r} u_{1}-\left|u_{2}\right|^{r} u_{2}\right\|_{H^{1}(M)} d s \\
& \leq C T\left\|u_{1}-u_{2}\right\|_{X_{T}}\left(\left\|u_{1}\right\|_{X_{T}}^{r}+\left\|u_{2}\right\|_{X_{T}}^{r}\right) .
\end{aligned}
$$

Let $A \equiv\|v\|_{X_{T}}+\|z\|_{L^{1}\left(0, T ; H^{1}(\Omega)\right)}$. Let also $R>0$ and $T>0$ be such that $A+C T R^{r+1}<R$; for example, after setting $R=2 A$, we can pick $T$ small enough such that $C T A^{r}<2^{-r-1}$. With these particular 
parameters, we see that $\Psi$ is a contractive mapping on $C l\left(B_{R}\left(0 ; X_{T}\right)\right)$, which is a closed and therefore complete subspace of $X_{T}$. By the contraction mapping theorem, we conclude that $\Psi$ has a unique fixed point in $C l\left(B_{R}\left(0 ; X_{T}\right)\right)$.

Note that no Strichartz estimate is needed in the one dimensional case to prove that $\Psi$ is a contraction.

\subsubsection{Two dimensional domains}

Again, we start with the $H^{1}$ estimate of $\Psi(u)$.

$$
\begin{aligned}
\|\Psi(u)\|_{H^{1}(M)} & =\left\|v(t)+e^{-i t \Delta}\left(u_{0}-v_{0}\right)-i \int_{0}^{t} e^{-i(t-s) \Delta} f_{z}(u) d s\right\|_{H^{1}(M)} \\
& \leq\|v(t)\|_{H^{1}(M)}+\left\|e^{-i t \Delta}\left(u_{0}-v_{0}\right)\right\|_{H^{1}(M)}+\int_{0}^{T}\left\|e^{-i(t-s) \Delta} f_{z}(u)\right\|_{H^{1}(M)} d s \\
& \leq\|v\|_{C\left([0, T] ; H^{1}(M)\right)}+\left\|u_{0}-v_{0}\right\|_{H^{1}(M)}+\int_{0}^{T}\left\||u|^{r} u-z\right\|_{H^{1}(M)} d s \\
& \leq\|v\|_{C\left([0, T] ; H^{1}(M)\right)}+\left\|u_{0}-v_{0}\right\|_{H^{1}(M)}+\|z\|_{L^{1}\left(0, T ; H^{1}(\Omega)\right)}+\int_{0}^{T}\left\||u|^{r} u\right\|_{H^{1}(M)} d s \\
& \leq\|v\|_{C\left([0, T] ; H^{1}(M)\right)}+\left\|u_{0}-v_{0}\right\|_{H^{1}(M)}+\|z\|_{L^{1}\left(0, T ; H^{1}(\Omega)\right)}+C_{r, p, T}\|u\|_{X_{T}}^{r+1} .
\end{aligned}
$$

In the above estimate, the second inequality follows by the conservation of the Schrödinger flow, and the last inequality follows by Lemma 3.2. Now, we estimate the $L^{p}\left(0, T ; L_{\infty}(M)\right)$ norm of $\Psi(u)$.

$$
\begin{aligned}
\|\Psi(u)\|_{L^{p}\left(0, T ; L^{\infty}(M)\right)}= & \left\|v(t)+e^{-i(t-s) \Delta}\left(u_{0}-v_{0}\right)+i \int_{0}^{t} e^{-i(t-s) \Delta} f_{z}(u) d s\right\|_{L^{p}\left(0, T ; L^{\infty}(M)\right)} \\
\leq & \|v\|_{L^{p}\left(0, T ; L^{\infty}(M)\right)}+\left\|e^{-i t \Delta}\left(u_{0}-v_{0}\right)\right\|_{L^{p}\left(0, T ; L^{\infty}(M)\right)} \\
& +\int_{0}^{T}\left\|e^{-i(t-s) \Delta} f_{z}(u)\right\|_{L^{p}\left(0, T ; L^{\infty}(M)\right)} d s \\
\leq & C\|v\|_{L^{p}\left(0, T ; H^{1+\epsilon}(M)\right)}+C\left\|u_{0}-v_{0}\right\|_{H^{1}(M)}+C \int_{0}^{T}\left\|\left.u\right|^{r} u-z\right\|_{H^{1}(M)} d s \\
\leq & C\|v\|_{C\left([0, T] ; H^{1+\epsilon}(M)\right)}+C\left\|u_{0}-v_{0}\right\|_{H^{1}(M)}+C\|z\|_{L^{1}\left(0, T ; H^{1}(\Omega)\right)} \\
& +C \int_{0}^{T}\left\||u|^{r} u\right\|_{H^{1}(M)} d s \\
\leq & C\|v\|_{C\left([0, T] ; H^{1+\epsilon}(M)\right)}+C\left\|u_{0}-v_{0}\right\|_{H^{1}(M)}+C\|z\|_{L^{1}\left(0, T ; H^{1}(\Omega)\right)} \\
& +C C_{r, p, T}\|u\|_{X_{T}}^{r+1} \cdot
\end{aligned}
$$

In (27), the second inequality follows by the Strichartz estimate (3), and the last inequality follows by (14).

Now, we want to get a contractive estimate for $\Psi$, as we did in the one dimensional case. We start with the $H^{1}$ estimate of the difference between images of two elements $u_{1}, u_{2} \in X_{T}$. 


$$
\begin{aligned}
\left\|\Psi\left(u_{1}\right)-\Psi\left(u_{2}\right)\right\|_{H^{1}(M)} & =\left\|\int_{0}^{t} e^{-i(t-s) \Delta}\left(\left|u_{1}\right|^{r} u_{1}-\left|u_{2}\right|^{r} u_{2}\right) d s\right\|_{H^{1}(M)} \\
& \leq \int_{0}^{T}\left\|e^{-i(t-s) \Delta}\left(\left|u_{1}\right|^{r} u_{1}-\left|u_{2}\right|^{r} u_{2}\right)\right\|_{H^{1}(M)} d s \\
& =\int_{0}^{T}\left\|\left|u_{1}\right|^{r} u_{1}-\left|u_{2}\right|^{r} u_{2}\right\|_{H^{1}(M)} d s .
\end{aligned}
$$

By using Lemma 3.3 and the general Hölder's inequality, we obtain

$$
\begin{aligned}
& \left\|\Psi\left(u_{1}\right)-\Psi\left(u_{2}\right)\right\|_{H^{1}(M)} \\
& \leq C \int_{0}^{T}\left\|u_{1}-u_{2}\right\|_{H_{0}^{1}(M)}\left(\left\|u_{1}\right\|_{L^{\infty}(M)}+\left\|u_{2}\right\|_{L^{\infty}(M)}\right)^{r} d t \\
& \quad+C \int_{0}^{T}\left\|u_{1}-u_{2}\right\|_{L^{\infty}(M)}\left(\left\|u_{1}\right\|_{L^{\infty}(M)}+\left\|u_{2}\right\|_{L^{\infty}(M)}\right)^{r-1}\left(\left\|u_{1}\right\|_{H^{1}(M)}+\left\|u_{2}\right\|_{H^{1}(M)}\right) d t \\
& \leq C T^{\frac{p-r}{p}}\left\|u_{1}-u_{2}\right\|_{X_{T}}\left(\left\|u_{1}\right\|_{X_{T}}^{r}+\left\|u_{2}\right\|_{X_{T}}^{r}\right) .
\end{aligned}
$$

Secondly, we estimate the $L^{p}\left(0, T ; L^{\infty}(M)\right)$ norm of the difference of two elements $\Psi\left(u_{1}\right)$ and $\Psi\left(u_{2}\right)$ where $u_{1}, u_{2} \in X_{T}$.

$$
\begin{aligned}
\left\|\Psi\left(u_{1}\right)-\Psi\left(u_{2}\right)\right\|_{L^{p}\left(0, T ; L^{\infty}(M)\right)} & =\left\|\int_{0}^{t} e^{-i(t-s) \Delta}\left(\left|u_{1}\right|^{r} u_{1}-\left|u_{2}\right|^{r} u_{2}\right) d s\right\|_{L^{p}\left(0, T ; L^{\infty}(M)\right)} \\
& \leq \int_{0}^{T}\left\|e^{-i(t-s) \Delta}\left(\left|u_{1}\right|^{r} u_{1}-\left|u_{2}\right|^{r} u_{2}\right)\right\|_{L^{p}\left(0, T ; L^{\infty}(M)\right)} d s \\
& \leq C \int_{0}^{T}\left\|\left|u_{1}\right|^{r} u_{1}-\left|u_{2}\right|^{r} u_{2}\right\|_{H^{1}(M)} d s
\end{aligned}
$$

where the last inequality follows by the Strichartz estimate (3). We estimate the last term in (30) as in (29) and get

$$
\left\|\Psi\left(u_{1}\right)-\Psi\left(u_{2}\right)\right\|_{L^{p}\left(0, T ; L^{\infty}(M)\right)} \leq C T^{\frac{p-r}{p}}\left\|u_{1}-u_{2}\right\|_{X_{T}}\left(\left\|u_{1}\right\|_{X_{T}}^{r}+\left\|u_{2}\right\|_{X_{T}}^{r}\right) .
$$

Let $A \equiv\|v\|_{C\left([0, T] ; H^{1}(M)\right)}+C\left\|u_{0}-v_{0}\right\|_{H^{1}(M)}+C\|z\|_{L^{1}\left(0, T ; H_{1}(\Omega)\right)}$. Let also $R>0$ and $T>0$ be such that $A+\left(T^{\frac{p-r-1}{p}}+(r+1) T^{\frac{p-r}{p}}\right) R^{r+1}<R$; for example choose $R=2 A$ and $T$ small enough. Choosing $T$ even smaller, we see that $\Psi$ becomes a contractive mapping on $C l\left(B_{R}\left(0 ; X_{T}\right)\right)$, which is a closed and therefore complete subspace of $X_{T}$. Therefore, it has a unique fixed point in $C l\left(B_{R}\left(0 ; X_{T}\right)\right)$.

Remark 6. Note that local solutions constructed by Strichartz estimates were unique in the subspace $B\left(0, R ; X_{T}\right)$. This does not immediately give uniqueness in $X_{T}$. However, we can utilize the fact that they are $L^{\infty}$ in space to prove uniqueness in $X_{T}$, as in the following lemma. 
Lemma 3.4. Local solutions in $X_{T}$ are unique.

Proof. Let $u_{1}$ and $u_{2}$ be two solutions of (1) in $X_{T}$, and set $w \equiv u_{1}-u_{2}$. Then $w$ satisfies the following initial boundary value problem.

$$
\begin{cases}D_{t} w+\Delta w+\lambda\left(\left|u_{1}\right|^{r} u_{1}-\left|u_{2}\right|^{r} u_{2}\right)=0 & \text { in } Q=(0, T) \times M \\ w(0)=0 & \text { in } M ; \\ B w=0 & \text { in } \Sigma=(0, T) \times \partial M .\end{cases}
$$

Multiplying (32) by $\bar{w}$, integrating over $M$ and taking the imaginary parts, one gets

$$
\begin{aligned}
\frac{1}{2} \frac{d}{d t}\|w\|_{L_{2}(M)}^{2} & =\lambda \operatorname{Im} \int_{M}\left(\left|u_{1}\right|^{r} u_{1}-\left|u_{2}\right|^{r} u_{2}\right) \bar{w} d x \\
& \leq C \int_{M}\left|u_{1}-u_{2}\right|\left(\left|u_{1}\right|^{r}+\left|u_{2}\right|^{r}\right)|w| d x \leq C\left(\left\|u_{1}\right\|_{L^{\infty}(M)}^{r}+\left\|u_{2}\right\|_{L^{\infty}(M)}^{r}\right)\|w\|_{L_{2}(M)}^{2}
\end{aligned}
$$

Upon integration in time and using Hölder's inequality, we get

$$
\|w\|_{L_{2}(M)}^{\frac{2 p}{p-r}} \leq C\left(\left\|u_{1}\right\|_{L^{p}\left(0, T ; L^{\infty}(M)\right)}+\left\|u_{2}\right\|_{L^{p}\left(0, T ; L^{\infty}(M)\right)}\right)^{\frac{r p}{p-r}} \int_{0}^{T}\|w\|_{L_{2}(M)}^{\frac{2 p}{p-r}} d s,
$$

which implies that $w \equiv 0$.

\subsection{Local stability}

Proof of Theorem 1.2. Let's consider the case (i) of Theorem 1.1. Other cases are proved similarly or more easily and omitted here. Let $u_{1}, u_{2}$ be two solutions with initial-boundary data $\left(u_{1}^{0}, h_{1}\right)$ and $\left(u_{2}^{0}, h_{2}\right)$, respectively. Since we know that the linear problem has stability, it is enough to prove that $\left\|u_{1}-u_{2}\right\|_{C\left([0, T] ; H^{1}(M)\right)}$ is controlled by the differences between initial data and also between solutions of associated linear problems. Indeed,

$$
\begin{aligned}
\left\|u_{1}-u_{2}\right\|_{C\left([0, T] ; H^{1}(M)\right) \leq} & \left\|u_{1}-u_{2}\right\|_{X_{T}} \leq C\left\|v_{1}-v_{2}\right\|_{C\left([0, T] ; H^{1+\epsilon}(M)\right)} \\
& +C\left\|u_{1}^{0}-u_{2}^{0}\right\|_{H^{1}(M)}+C T^{\frac{p-r}{p}}\left\|u_{1}-u_{2}\right\|_{X_{T}}\left(\left\|u_{1}\right\|_{X_{T}}^{r}+\left\|u_{2}\right\|_{X_{T}}^{r}\right) .
\end{aligned}
$$

Choosing $R$, which depends on $u_{0}$ and $h$ (i.e., on the bounded set $O$ ), as in the proof of the local existence, and $T$ accordingly small enough, we obtain

$$
\left\|u_{1}-u_{2}\right\|_{C\left([0, T] ; H^{1}(M)\right)} \leq C\left\|v_{1}-v_{2}\right\|_{C\left([0, T] ; H^{1+\epsilon}(M)\right)}+C\left\|u_{1}^{0}-u_{2}^{0}\right\|_{H^{1}(M)} .
$$

Now, the result follows by the continuous dependence on data for the linear problem.

\subsection{Control of the $H^{1}$-norm}

The passage from a local solution to a global one can be achieved if one has a uniform $H^{1}$-bound in time. It is well known that solutions of the nonlinear Schrödinger equation with homogeneous Dirichlet or Neumann boundary conditions have $L^{2}$ and $H^{1}$ conservation laws. Unfortunately, these conservation laws are no longer valid in the case of inhomogeneous boundary conditions. One can see this with a straightforward 
calculation. For example, suppose $u$ is a smooth solution of (1) for a moment. Then, multiplying (1) with $\bar{u}$ in a formal sense, integrating over $M$ and taking the imaginary parts, one can see that the mass changes with respect to the law

$$
\frac{1}{2} \frac{d}{d t}\|u\|_{L_{2}(M)}^{2}=\operatorname{Im} \int_{\partial M} \frac{\partial u}{\partial n} \bar{u} d \Gamma
$$

Similarly, multiplying (1) with $\bar{u}_{t}$, integrating over $M$ and taking the real parts, one gets

$$
\frac{1}{2} \frac{d}{d t}\left(\|\nabla u\|_{L_{2}(M)}^{2}-\frac{2 \lambda}{r+2}\|u\|_{L_{r+2}(M)}^{r+2}\right)=\operatorname{Re} \int_{\partial M} \frac{\partial u}{\partial n} \bar{u}_{t} d \Gamma .
$$

For the Neumann problem, one may alternatively write (36) as

$$
\frac{1}{2} \frac{d}{d t}\left(\|\nabla u\|_{L_{2}(M)}^{2}-\frac{2 \lambda}{r+2}\|u\|_{L^{r+2}(M)}^{r+2}\right)=\frac{d}{d t}\left(\operatorname{Re} \int_{\partial M} h \bar{u} d \Gamma\right)-\operatorname{Re} \int_{\partial M} h_{t} \bar{u} d \Gamma .
$$

It is apparent from the identities (35)-(37) that in order to control the $H^{1}$ norm, one needs to bound the trace of the directional derivative and the solution in appropriate spaces for Dirichlet and Neumann problems, respectively. See for example [16] for the treatment of the defocusing problem with Dirichlet force, [17] for the focusing problem with Dirichlet force and [18] for both focusing and defocusing problems with Neumann force. The situation is relatively easier for the Neumann problem since one can use Sobolev trace inequalities. The Dirichlet problem is more delicate and requires a special multiplier.

In order to use the multiplier method directly on a partial differential equation, one needs sufficient regularity of the solution. The classical approach is first constructing approximate solutions and obtaining uniform estimates on those solutions. Finally, a passage to the limit gives information about the original solution. For example, one may use the monotone operator theory $[16,17]$ or perturbed Galerkin's approach [18] to obtain sufficiently smooth approximate solutions for (1).

Recall that perturbed Galerkin approximations with smooth data $\left(u_{0 N}, h_{N}\right)$ (which converge to $\left(u_{0}, h\right)$ in the spaces given in Theorem 1.1) for (1) are defined by $u_{N} \equiv v_{N}+w_{N}$, where $v_{N}$ solves the linear problem (5) (Dirichlet) or (7) (Neumann) and $w_{N} \equiv \sum_{n=1}^{N} d_{n}^{N}(t) \varphi_{n}$ is a Galerkin approximation for the homogenized nonlinear problem (8). The time dependent coefficients $d_{n}^{N}$ are extracted from the $N \times N$ system of first order ordinary differential equations:

$$
\left\{\begin{array}{l}
i \dot{d}_{n}^{N}+\lambda_{n} d_{n}^{N}+f_{n}^{N}(t)=0, \quad n \leq N \\
d_{n}^{N}(0)=\alpha_{n}^{N}
\end{array}\right.
$$

where $f_{n}^{N}=\left(f_{v_{N}, z_{N}}\left(w_{N}\right), \varphi_{n}\right)_{L_{2}(M)}, \alpha_{n}^{N}=\left(v_{0 N}-u_{0 N}, \varphi_{n}\right)_{L^{2}(M)},\left(\varphi_{n}\right)_{n=1}^{\infty}$ are the eigenfunctions of the Laplacian (with homogeneous Dirichlet or Neumann boundary conditions) associated with the eigenvalues $\lambda_{n}$.

Observe that approximate solutions satisfy mass and energy identities (35)-(37). These will be sufficient to treat Neumann type forces, but for Dirichlet types force we will need the following lemma.

Lemma 3.5. Let $q \in\left[C^{1}(\bar{\Omega})\right]^{n}$ be a real vector field with the property $\left.q\right|_{\Gamma}=n$, and let $Q(x)$ be the $d \times d$ matrix with entries $Q_{i j}=\frac{\partial q_{i}}{\partial x_{j}}$. Then, the following identity holds true.

$$
\frac{d}{d t} \operatorname{Im} \int_{M} u_{N}\left(q \cdot \nabla \bar{u}_{N}\right) d x=\operatorname{Im} \int_{\Gamma} h_{N} \bar{h}_{N t} d \Gamma+2 \operatorname{Re} \int_{M}\left(Q \nabla u_{N}\right) \cdot \nabla \bar{u}_{N} d x
$$




$$
\begin{aligned}
& +\left\|\nabla_{A} h_{N}\right\|_{L^{2}(\Gamma)}^{2}-\left\|\nabla u_{N} \cdot n\right\|_{L^{2}(\Gamma)}^{2}+\operatorname{Re} \int_{M}\left(\nabla(\operatorname{div} q) \cdot \nabla u_{N}\right) \bar{u}_{N} d x \\
& -\operatorname{Re} \int_{\Gamma}\left(\nabla u_{N} \cdot n\right) \bar{h}_{N} \operatorname{div} q d \Gamma-\frac{2 \lambda}{r+2} \int_{\Gamma}\left|h_{N}\right|^{r+2} d \Gamma-\frac{\lambda r}{r+2} \int_{M}(\operatorname{div} q)\left|u_{N}\right|^{r+2} d x .
\end{aligned}
$$

Proof. The proof is classical and we use the special multiplier $q \cdot \nabla \bar{u}_{N}$. We start with the following identity.

$$
\frac{d}{d t} \operatorname{Im} \int_{M} u_{N}\left(q \cdot \nabla \bar{u}_{N}\right) d x=\operatorname{Im} \int_{M} u_{N t}\left(q \cdot \nabla \bar{u}_{N}\right) d x+\operatorname{Im} \int_{M} u_{N}\left(q \cdot \nabla \bar{u}_{N t}\right) d x .
$$

Using the divergence theorem and $\left.q\right|_{\Gamma}=n$, one gets

$$
\operatorname{Im} \int_{M} u_{N} q \cdot \nabla \bar{u}_{N t} d x=\operatorname{Im} \int_{\Gamma} h_{N} \bar{h}_{N t} d \Gamma-\operatorname{Im} \int_{M}\left(q \cdot \nabla u_{N}\right) \bar{u}_{N t} d x-\operatorname{Im} \int_{M} \operatorname{div}(q) u_{N} \bar{u}_{N t} d x .
$$

Using (38), the linear equation and the divergence theorem,

$$
\begin{aligned}
-\operatorname{Im} \int_{M} \operatorname{div}(q) u_{N} \bar{u}_{N t} d x= & \int_{M} \operatorname{div}(q) \nabla u_{N} \cdot \nabla \bar{u}_{N} d x+\operatorname{Re} \int_{M}\left(\nabla(\operatorname{div}(q)) \cdot \nabla u_{N}\right) \bar{u}_{N} d x \\
& -\lambda \int_{M}\left|u_{N}\right|^{r+2} \operatorname{div}(q) d x-\operatorname{Re} \int_{\Gamma}\left(\nabla u_{N} \cdot n\right) \bar{h}_{N} \operatorname{div}(q) d \Gamma .
\end{aligned}
$$

Using (38) and the linear equation again,

$$
-\operatorname{Im} \int_{M}\left(q \cdot \nabla u_{N}\right) \bar{u}_{N t} d x=-\operatorname{Re} \int_{M} \Delta u_{N}\left(q \cdot \nabla \bar{u}_{N}\right) d x-\lambda \operatorname{Re} \int_{M}\left|u_{N}\right|^{r} u_{N} q \cdot \nabla \bar{u}_{N} d x
$$

where

$$
\begin{aligned}
&-\operatorname{Re} \int_{M} \Delta u_{N}\left(q \cdot \nabla \bar{u}_{N}\right) d x= \operatorname{Re} \int_{M} \nabla u_{N} \cdot \nabla\left(q \cdot \nabla \bar{u}_{N}\right) d x-\left\|\nabla u_{N} \cdot n\right\|_{L^{2}(\Gamma)}^{2} \\
&= \operatorname{Re} \int_{M}\left(Q \nabla u_{N}\right) \cdot \nabla \bar{u}_{N} d x+\frac{1}{2} \int_{M} q \cdot \nabla\left(\left|\nabla u_{N}\right|^{2}\right) d x-\left\|\nabla u_{N} \cdot n\right\|_{L^{2}(\Gamma)}^{2} \\
&= \operatorname{Re} \int_{M}\left(Q \nabla u_{N}\right) \cdot \nabla \bar{u}_{N} d x-\frac{1}{2} \int_{M} \operatorname{div}(q) \nabla u_{N} \cdot \nabla \bar{u}_{N} d x \\
&+\frac{1}{2}\left\|\nabla u_{N}\right\|_{L^{2}(\Gamma)}^{2}-\left\|\nabla u_{N} \cdot n\right\|_{L^{2}(\Gamma)}^{2} \cdot \\
&-\lambda \operatorname{Re} \int_{M}\left|u_{N}\right|^{r} u_{N}\left(h_{N} \cdot \nabla \bar{u}_{N}\right) d x=-\frac{2 \lambda}{r+2} \int_{\Gamma}\left|h_{N}\right|^{r+2}(q \cdot n) d \Gamma+\frac{2 \lambda}{r+2} \int_{M} \operatorname{div}(q)\left|u_{N}\right|^{r+2} d x .
\end{aligned}
$$

Observe that the tangential component of $\nabla w_{N}$ on $\Gamma$ is zero since $w_{N} \equiv 0$ on $\partial M$. Moreover, $\nabla w_{N}=$ $\frac{\partial w_{N}}{\partial n} n$. Hence,

$$
\nabla u_{N} \cdot A=\nabla w_{N} \cdot A+\nabla v_{N} \cdot A=\nabla v_{N} \cdot A
$$


where $A$ is the unit tangential vector. Using (46), we obtain

$$
\left|\nabla u_{N}\right|^{2}=\left|\nabla u_{N} \cdot n\right|^{2}+\left|\nabla u_{N} \cdot A\right|^{2}=\left|\nabla u_{N} \cdot n\right|^{2}+\left|\nabla_{A} h_{N}\right|^{2} .
$$

Now (39) follows by (40)-(45) and (47).

\subsubsection{Dirichlet problem with defocusing source}

We set

$$
E_{N} \equiv\left\|u_{N}\right\|_{H^{1}(M)}^{2}+\frac{2}{r+2}\left\|u_{N}\right\|_{L^{r+2}(M)}^{r+2}
$$

and

$$
H_{N} \equiv \int_{0}^{t}\left(\left\|h_{N}\right\|_{L^{2}(\Gamma)}^{2}+\left\|h_{N t}\right\|_{L^{2}(\Gamma)}^{2}+\left\|\left.\nabla_{A} h_{N}\right|_{L^{2}(\Gamma)} ^{2}+\right\| h_{N} \|_{L^{r+2}(\Gamma)}^{r+2}\right) d s .
$$

(35) and (36) imply

$$
E_{N} \leq C+\epsilon \int_{0}^{t}\left\|\nabla u_{N} \cdot n\right\|_{L^{2}(\Gamma)}^{2} d s
$$

with $\epsilon>0$ being a sufficiently small fixed parameter whose value can be fixed to an even smaller number in further calculations.

Remark 7. In (48) and in what follows, $C=C\left(M, r,\left\|u_{0 N}\right\|_{H^{1}(M)}, H_{N}, T, \epsilon\right)$ is a generic constant depending on the fixed parameters.

Now, using (39), we have

$$
\int_{0}^{t}\left\|\nabla u_{N} \cdot n\right\|_{L^{2}(\Gamma)}^{2} d s \leq C+C E_{N}+C \int_{0}^{t} E_{N} d s
$$

Combining (48) and (49), we get

$$
E_{N} \leq C+\epsilon \int_{0}^{t} E_{N} d s
$$

Applying Gronwall's inequality to (50), we get

$$
E_{N} \leq C e^{\epsilon T}
$$

\subsubsection{Dirichlet problem with focusing source}

We set $B_{N}=\int_{0}^{t}\left\|\nabla u_{N} \cdot n\right\|_{L^{2}(\Gamma)}^{2} d s$, and $D_{N} \equiv \sup _{[0, t]}\left\{\left\|u_{N}\right\|_{H^{1}(M)}^{2}\right\}$. By (35) and (36), the following estimates follow.

$$
\left\|u_{N}\right\|_{L^{2}(M)}^{2} \leq\left\|u_{0 N}\right\|_{L^{2}(M)}^{2}+2 \sqrt{H_{N} B_{N}}
$$


and

$$
\left\|\nabla u_{N}\right\|_{L^{2}(M)}^{2} \leq\left\|\nabla u_{0 N}\right\|_{L^{2}(M)}^{2}+\frac{1}{\epsilon} H_{N}+\epsilon B_{N}+C\left\|u_{N}\right\|_{L^{r+2}(M)}^{r+2} \leq C+\epsilon B_{N}+C\left\|u_{N}\right\|_{L^{r+2}(M)}^{r+2} .
$$

By the Gagliardo-Nirenberg inequality:

$$
\left\|u_{N}\right\|_{L^{r+2}(M)}^{r+2} \leq C\left\|\nabla u_{N}\right\|_{L^{2}(M)}^{\theta(r+2)}\left\|u_{N}\right\|_{L^{2}(M)}^{(1-\theta)(r+2)}+C\left\|u_{N}\right\|_{L^{2}(M)}
$$

where $\frac{1}{r+2}=\frac{1}{2}-\frac{\theta}{d}$. Under our assumption $0<r<\frac{4}{d+2}$, we have $\theta(r+2)=\frac{d r}{2}<2$ and $(1-\theta)(r+2)=$ $r+2-\frac{d r}{2}=\frac{2 r+4-d r}{2}$. Note that also $1 /\left(1-\frac{d r}{4}\right)=\frac{4}{4-d r}$ and $\mu:=\frac{2 r+4-d r}{2} \frac{4}{4-d r}=\frac{4 r}{4-d r}+2<4$. Therefore, by Young's inequality, the right hand side of (54) is bounded by

$$
\epsilon\left\|\nabla u_{N}\right\|_{L^{2}(M)}^{2}+C\left\|u_{N}\right\|_{L^{2}(M)}^{\mu}+\frac{\epsilon}{2}\left\|u_{N}\right\|_{L^{2}(M)}^{4}+C \leq \epsilon\left\|u_{N}\right\|_{H^{1}(M)}^{2}+\epsilon\left\|u_{N}\right\|_{L_{2}(M)}^{4}+C .
$$

Using (39), we have

$$
B_{N} \leq C+C D_{N}+C \int_{0}^{t} D_{N} d s+C \int_{0}^{t}\left\|u_{N}\right\|_{L^{r+2}(M)}^{r+2} d s
$$

By (52) and (55),

$$
B_{N} \leq C+C D_{N}+C \int_{0}^{t} D_{N} d s+\epsilon \int_{0}^{t} B_{N} d s
$$

By Gronwall's inequality,

$$
B_{N} \leq C+C D_{N}+C \int_{0}^{t} D_{N} d s
$$

On the other hand,

$$
D_{N} \leq C_{N}+2 \epsilon B_{N}+C\left\|u_{N}\right\|_{r+2}^{r+2} .
$$

Again by using (52) and (55),

$$
D_{N} \leq C+\epsilon \int_{0}^{t} D_{N} d s
$$

Now, the boundedness of $D_{N}$ follows by Gronwall's inequality and we have $D_{N} \leq C e^{\epsilon T}$.

\subsubsection{Neumann problem with defocusing source}

Let us set

$$
H_{N} \equiv \sup _{[0, t]}\left(\left\|h_{N}\right\|_{L^{2}(\Gamma)}^{2}+\left\|h_{N t}\right\|_{L^{2}(\Gamma)}^{2}\right) d s
$$


Now, we use (35) and the Sobolev trace inequality to get the inequality

$$
\left\|u_{N}\right\|_{L^{2}(M)}^{2} \leq C+\epsilon \int_{0}^{t}\left\|u_{N}\right\|_{H^{1}(M)}^{2} d s .
$$

Similarly, by (37), we obtain

$$
\left\|\nabla u_{N}\right\|_{L^{2}(M)}^{2}+\frac{2}{r+2}\left\|u_{N}\right\|_{L^{r+2}(M)}^{r+2} \leq C+\epsilon\left\|u_{N}\right\|_{H^{1}(M)}^{2}+\epsilon \int_{0}^{t}\left\|u_{N}\right\|_{H^{1}(M)}^{2} d s .
$$

Combining (59) and (60), we obtain

$$
E_{N} \leq C+\epsilon \int_{0}^{t} E_{N} d s
$$

Again by Gronwall's inequality, we deduce that

$$
E_{N} \leq C e^{\epsilon T}
$$

\subsubsection{Neumann problem with focusing source}

From (35), we compute

$$
\left\|u_{N}\right\|_{L_{2}(M)}^{4} \leq C+C\left\|u_{N}\right\|_{H^{1}(M)}^{2} .
$$

Now, combining (59) and (60),

$$
\left\|u_{N}\right\|_{H^{1}(M)}^{2} \leq C+\epsilon \int_{0}^{t}\left\|u_{N}\right\|_{H^{1}(M)}^{2} d s+C\left\|u_{N}\right\|_{L^{r+2}(M)}^{r+2} .
$$

Using (62) and (55), we have

$$
\left\|u_{N}\right\|_{H^{1}(M)}^{2} \leq C+\epsilon \int_{0}^{t}\left\|u_{N}\right\|_{H^{1}(M)}^{2} d s
$$

Applying Gronwall's inequality to (64), we obtain the desired boundedness:

$$
\left\|u_{N}\right\|_{H^{1}(M)}^{2} \leq C e^{\epsilon T} .
$$

\subsubsection{Passage to the limit}

By the classical compactness method, there exists a weak $H^{1}$-solution $u$ of (1) such that a subsequence of $u_{N}$ (still denoted $u_{N}$ ) converges to $u$ weakly in $H^{1}$; see [16-18] for more details. We will need two convergence properties of $u_{N}$ 's in the next section. For the Dirichlet problem, $\frac{\partial u_{N}}{\partial n} \rightarrow \frac{\partial u}{\partial n}$ in $L^{2}(\Sigma)$ and for the Neumann problem $u_{N} \rightarrow u$ in $L^{2}(\Sigma)$.

\section{Remark 8.}

(i) Uniform boundedness of $u$ follows by the weak lower semicontinuity of the $H^{1}$-norm. By the uniform boundedness of the $H^{1}$ norm we can extend local solutions to any arbitrary time interval [0,T]. 
(ii) Observe that for the Dirichlet problem we in particular obtain $\frac{\partial u}{\partial n} \in L^{2}(\Sigma)$. Formally speaking, this is $\frac{1}{2}$ derivatives better than the classical trace theory would suggest. Some authors call this gain of the regularity the hidden regularity.

\subsection{Continuity of a unique and essentially bounded solution}

In this section, we use similar arguments to [5, Theorem 3.3.9] to pass from a weak $H^{1}$-solution to a strong $H^{1}$-solution. We combine uniqueness results with $H^{1}$-energy inequalities and the fact that the weak $H^{1}$-solution belongs to the function space $L^{\infty}\left(0, T ; H^{1}(M)\right) \cap W^{1, \infty}\left(0, T ; H^{-1}(M)\right)$ to prove the continuity of solutions in time, and therefore the existence of a unique strong $H^{1}$-solution follows. We prove the following lemma which is true for any dimension.

In order prove Theorem 1.3, we first prove the following convergence properties.

Lemma 3.6. Let $u_{N}$ be as in Section 3.3.5. Then, for the Dirichlet problem

$$
\lim _{N \rightarrow \infty} \int_{0}^{t}\left\langle\frac{\partial u_{N}}{\partial n}, h_{N}+i h_{N t}\right\rangle_{\Gamma} d s=\int_{0}^{t}\left\langle\frac{\partial u}{\partial n}, h+i h_{t}\right\rangle_{\Gamma} d s
$$

and for the Neumann problem

$$
\lim _{N \rightarrow \infty} \int_{0}^{t}\left\langle h_{N}+i h_{N t}, u_{N}\right\rangle_{\Gamma} d s=\int_{0}^{t}\left\langle h+i h_{t}, u\right\rangle_{\Gamma} d s
$$

and

$$
\lim _{N \rightarrow \infty}\left\langle h_{N}, i u_{N}\right\rangle_{\Gamma}=\langle h, i u\rangle_{\Gamma}
$$

Remark 9. In the above lemma, $\langle\cdot, \cdot\rangle_{\Gamma}$ denotes the $L^{2}$ inner product on $\Gamma$.

Proof of Lemma 3.6. Let's first consider the Dirichlet problem. Then,

$$
\begin{aligned}
& \left|\int_{0}^{t}\left\langle\frac{\partial u_{N}}{\partial n}, h_{N}+i h_{N t}\right\rangle_{\Gamma} d s-\int_{0}^{t}\left\langle\frac{\partial u}{\partial n}, h+i h_{t}\right\rangle_{\Gamma} d s\right| \\
& \quad=\left|\int_{0}^{t}\left\langle\frac{\partial u_{N}}{\partial n}, h_{N}+i h_{N t}-h-i h_{t}\right\rangle_{\Gamma} d s+\int_{0}^{t}\left\langle\frac{\partial u_{N}}{\partial n}-\frac{\partial u}{\partial n}, h+i h_{t}\right\rangle_{\Gamma} d s\right| \\
& \quad \leq\left\|\frac{\partial u_{N}}{\partial n}\right\|_{L^{2}(\Sigma)}\left\|h_{N}+i h_{N t}-h-i h_{t}\right\|_{L^{2}(\Sigma)}+\left|\int_{0}^{t}\left\langle\frac{\partial u_{N}}{\partial n}-\frac{\partial u}{\partial n}, h+i h_{t}\right\rangle_{\Gamma} d s\right| .
\end{aligned}
$$

Now, by boundedness of $\left\|\frac{\partial u_{N}}{\partial n}\right\|_{L^{2}(\Sigma)}$, convergence of $h_{N}$ to $h$ in $L^{2}(\Sigma)$, and weak convergence of $\frac{\partial u_{N}}{\partial n}$ to $\frac{\partial u}{\partial n}$ in $L^{2}(\Sigma)$, passing to the limit in (69), we obtain (66).

For the Neumann problem, we similarly check the following difference,

$$
\left|\int_{0}^{t}\left\langle h_{N}+i h_{N t}, u_{N}\right\rangle_{\Gamma} d s-\int_{0}^{t}\left\langle h+i h_{t}, u\right\rangle_{\Gamma} d s\right|
$$




$$
\begin{aligned}
& =\left|\int_{0}^{t}\left\langle h_{N}+i h_{N t}-h-i h_{t}, u_{N}\right\rangle_{\Gamma} d s+\int_{0}^{t}\left\langle h+i h_{t}, u_{N}-u\right\rangle_{\Gamma} d s\right| \\
& \leq\left\|h_{N}+i h_{N t}-h-i h_{t}\right\|_{L^{2}(\Sigma)}\left\|u_{N}\right\|_{L^{2}(\Sigma)}+\left|\int_{0}^{t}\left\langle h+i h_{t}, u_{N}-u\right\rangle_{\Gamma} d s\right| .
\end{aligned}
$$

Now, by Sobolev trace inequality $\left\|u_{N}\right\|_{L^{2}(\Gamma)} \leq C\left\|u_{N}\right\|_{H^{1}(M)}$, boundedness of $\left\|u_{N}\right\|_{H^{1}(M)}$, strong convergence of $h_{N}$ to $h$ in $L^{2}(\Sigma)$, and weak convergence of $u_{N}$ to $u$ in $L^{2}(\Sigma)$, we obtain (67).

Similarly, regarding (68), we write

$$
\begin{aligned}
\left|\left\langle h_{N}, i u_{N}\right\rangle_{\Gamma}-\langle h, i u\rangle_{\Gamma}\right| & =\left|\left\langle h_{N}-h, i u_{N}\right\rangle_{\Gamma}+\left\langle h, i u_{N}-i u\right\rangle_{\Gamma}\right| \\
& \leq\left\|h_{N}-h\right\|_{L^{2}(\Gamma)}|| u_{N} \|_{L^{2}(\Gamma)}+\left|\left\langle h, i u_{N}-i u\right\rangle_{\Gamma}\right| .
\end{aligned}
$$

Now, by the same arguments used for (67), we obtain (68).

Proof of Theorem 1.3. First, we observe that passing to the limit in (35), we have $\left\|u_{N}\right\|_{L^{2}(M)} \rightarrow\|u\|_{L^{2}(M)}$ uniformly on $[0, T]$, which implies that $u_{N} \rightarrow u$ in $C\left([0, T] ; L^{2}(M)\right)$. Now, using this, uniform boundedness of $u_{N}$ in $H^{1}(M)$, and Gagliardo-Nirenberg estimate, we obtain that $u_{N} \rightarrow u$ in $C\left([0, T] ; L^{r+2}(M)\right)$.

Now, for the Dirichlet problem we define

$$
E_{\mathbf{D}}(u(t))=\|u\|_{H^{1}(M)}^{2}-\frac{2 \lambda}{r+2}\|u\|_{L^{r+2}(M)}^{r+2}-2 \operatorname{Im} \int_{0}^{t}\left\langle\frac{\partial u}{\partial n}, h+i h_{t}\right\rangle_{\Gamma} d s
$$

whenever it makes sense.

Similarly, for the Neumann problem we define

$$
E_{\mathbf{N}}(u(t))=\|u\|_{H^{1}(M)}^{2}-\frac{2 \lambda}{r+2}\|u\|_{L^{r+2}(M)}^{r+2}-\left.2 \operatorname{Im}\langle h, i u\rangle_{\Gamma}\right|_{0} ^{t}-2 \operatorname{Im} \int_{0}^{t}\left\langle h+i h_{t}, u\right\rangle_{\Gamma} d s .
$$

Let $u_{N}$ be a perturbed Galerkin approximation, then

$$
\left.E_{\mathbf{D}}\left(u_{N}\right) \text { (or for the Neumann problem } E_{\mathbf{N}}\left(u_{N}\right)\right)=\left\|u_{0 N}\right\|_{H^{1}(M)}^{2}-\frac{2 \lambda}{r+2}\left\|u_{0 N}\right\|_{L^{r+2}(M)}^{r+2} .
$$

Using Lemma 3.6, we pass to liminf in (74) and get

$$
E_{\mathbf{D}}(u(t)) \leq\left\|u_{0}\right\|_{H^{1}(M)}^{2}-\frac{2 \lambda}{r+2}\left\|u_{0}\right\|_{L^{r+2}(M)}^{r+2} \equiv E\left(u_{0}\right)
$$

for the Dirichlet problem. Similarly,

$$
E_{\mathbf{N}}(u(t)) \leq\left\|u_{0}\right\|_{H^{1}(M)}^{2}-\frac{2 \lambda}{r+2}\left\|u_{0}\right\|_{L^{r+2}(M)}^{r+2} \equiv E\left(u_{0}\right)
$$

for the Neumann problem.

Now, referring to Dirichlet problem, let $\sigma, \tau \in[0, T], \sigma<\tau$ and let $v$ be a solution with $v(0)=u(\sigma)$ and $B v=h(t+\sigma)$. Then by uniqueness $v(t-\sigma)=u(t)$ and

$$
E_{\mathbf{D}}(v(\tau-\sigma))=E(u(\tau))-2 \operatorname{Im} \int_{\sigma}^{\tau}\left\langle\frac{\partial u}{\partial n}, h+i h_{t}\right\rangle_{\Gamma} d s \leq E(v(0))=E(u(\sigma)) .
$$


Similarly, let $w$ be a solution with $w(0)=u(\tau)$ and $B w=h(t+\tau)$. Then by uniqueness, $w(t-\tau)=u(t)$. Moreover, it is not difficult to see that the Schrödinger equation is time reversible and all the results that we have proved, are valid for negative times, too. Therefore,

$$
E_{\mathbf{D}}(w(\sigma-\tau))=E(u(\sigma))-2 \operatorname{Im} \int_{\tau}^{\sigma}\left\langle\frac{\partial u}{\partial n}, h+i h_{t}\right\rangle_{\Gamma} d s \leq E(w(0))=E(u(\tau)) .
$$

It follows that

$$
E(u(\tau))-E(u(\sigma))=2 \operatorname{Im} \int_{\sigma}^{\tau}\left\langle\frac{\partial u}{\partial n}, h+i h_{t}\right\rangle_{\Gamma} d s
$$

which implies $E(u(t))$ is continuous. The Neumann problem is treated in the same way, and we again get the continuity of $t \mapsto E(u(t))$. On the other hand, $u \in C^{0, \frac{1}{2}}\left([0, T] ; L^{2}(M)\right)$, see e.g., [5, Lemma 3.3.6] which implies that $t \mapsto\|u(t)\|_{L^{r+2}(M)}^{r+2}$ is continuous on $[0, T]$, see e.g., [5, Lemma 3.3.7]. It follows that $t \mapsto\|u(t)\|_{H^{1}(M)}$ is continuous on $[0, T]$. Therefore, $u \in C\left([0, T] ; H^{1}(M)\right) \cap C^{1}\left([0, T] ;\left(H^{1}(M)\right)^{\prime}\right)$.

\subsection{Subquadratic nonlinearities $(r<1)$}

Note that the fixed point method used in conjunction with Strichartz estimates works only for $r \geq 1$. However, we know that weak $H^{1}$-solutions exist for the defocusing problem for $r>0[16,19]$, and for the focusing problem for $r<\frac{4}{d+2}$ [17]. In the focusing case, for one and two dimensional domains, these correspond to $r<\frac{4}{3}$ and $r<1$, respectively. We can deal with the case $r<1$ using classical methods. In one dimension, we can use Sobolev's imbedding $L^{\infty}(M) \hookrightarrow H^{1}(M)$, and in dimension two, we can use Trudinger's inequality to prove uniqueness of weak $H^{1}$-solutions. Then, continuity in time and hence the existence of strong solutions follows by Theorem 1.3.

\subsubsection{Uniqueness in one dimension}

As in the proof of Lemma 3.4, we let $u_{1}$ and $u_{2}$ be two solutions, and set $w \equiv u_{1}-u_{2}$. Then, again

$$
\begin{aligned}
\frac{1}{2} \frac{d}{d t}\|w\|_{L_{2}(M)}^{2} & \leq\left.\int_{M}|| u_{1}\right|^{r} u_{1}-\left|u_{2}\right|^{r} u_{2}|| w \mid d x \\
& \leq C \int_{M}\left|u_{1}-u_{2}\right|\left(\left|u_{1}\right|^{r}+\left|u_{2}\right|^{r}\right)|w| d x \leq C\left(\left\|u_{1}\right\|_{L^{\infty}(M)}^{r}+\left\|u_{2}\right\|_{L^{\infty}(M)}^{r}\right)\|w\|_{L_{2}(M)}^{2} .
\end{aligned}
$$

Using $(75)$ and $w(0)=0$, we conclude that $w \equiv 0$.

\subsubsection{Uniqueness in two dimension}

We again set $w \equiv u_{1}-u_{2}$. We also set $q \equiv\left|u_{1}\right|+\left|u_{2}\right|$. (33) is still valid for $d=2$, from which we have

$$
\begin{aligned}
\frac{1}{2} \frac{d}{d t}\|w\|_{L_{2}(M)}^{2} & \leq\left. C \int_{M}|| u_{1}\right|^{r}+\left.\left|u_{2}\right|^{r}|| w\right|^{2} d x \leq C \int_{M} q^{r}|w|^{2} d x \\
& =C \int_{M} q^{r}|w|^{\delta}|w|^{2-\delta} d x \leq C\left(\int_{M}\left(q^{r}|w|^{\delta}\right)^{\frac{2}{\delta}} d x\right)^{\frac{\delta}{2}}\left(\int_{M}|w|^{2} d x\right)^{\frac{2-\delta}{2}} \\
& \leq C\|w\|_{L^{2}(M)}^{2-\delta}\|w\|_{L^{4}(M)}^{\delta}\left(\int_{M} q^{\frac{4 r}{\delta}} d x\right)^{\frac{\delta}{4}} .
\end{aligned}
$$


Trudinger's inequality implies that for $d=2$, given $q \in H^{1}(M)$, there are constants $\alpha, \beta>0$ such that $\int_{M} e^{\alpha q^{2}} d x \leq \beta$ where $\alpha$ and $\beta$ depend on $\|q\|_{H^{1}(M)}$.

$$
\begin{aligned}
\left(\int_{M} q^{\frac{4 r}{\delta}} d x\right)^{\frac{\delta}{4}} & \leq\left(\operatorname{vol}(M)^{\frac{\delta(2-r)}{8}}\right)\left(\int_{M} q^{\frac{8}{\delta}} d x\right)^{\frac{\delta r}{8}} \\
& \leq\left(\frac{4}{\alpha \delta}\right)^{\frac{r}{2}}\left(\operatorname{vol}(M)^{\frac{\delta(2-r)}{8}}\right)\left(\int_{M} e^{\alpha q^{2}-\frac{4}{\delta}} d x\right)^{\frac{\delta r}{8}} \leq C \delta^{-\frac{r}{2}}
\end{aligned}
$$

Using (76), (77), and the imbedding $L^{4}(M) \hookrightarrow H^{1}(M)$, we conclude that

$$
\frac{d}{d t}\|w\|_{L_{2}(M)}^{2} \leq C \delta^{-\frac{r}{2}}\|w\|_{L^{2}(M)}^{2-\delta} .
$$

Integrating this over a finite interval $[0, t]$, we get $\|w\|_{L_{2}(M)} \leq\left(C \delta^{1-\frac{r}{2}} T\right)^{\frac{2}{\delta}}$. Letting $\delta \rightarrow 0$, we obtain the desired result.

Remark 10. The argument above, which uses Trudinger's inequality, also works for $r \leq 2$, where the case $r=2$ has a trick! Indeed, for $r=2$, one first proves the desired result for some small time $t_{0}>0$ by choosing $t_{0}$ small enough so that $C \delta^{1-\frac{2}{2}} t_{0}=C t_{0}<1$. Then, repetition of the same argument gives the uniqueness on the entire time interval $[0, T]$.

\section{References}

[1] R. Anton, Strichartz inequalities for Lipschitz metrics on manifolds and nonlinear Schrödinger equation on domains, Bull. Soc. Math. France 136 (1) (2008) 27-65.

[2] C. Audiard, On the non-homogeneous boundary value problem for Schrödinger equations, Discrete Contin. Dyn. Syst. 33 (3) (2013) 3861-3884.

[3] M.D. Blair, H.F. Smith, C.D. Sogge, On Strichartz estimates for Schrödinger operators in compact manifolds with boundary, Proc. Amer. Math. Soc. 136 (1) (2008) 247-256.

[4] C. Bu, K. Tsutaya, C. Zhang, Nonlinear Schrödinger equation with inhomogeneous Dirichlet boundary data, J. Math. Phys. 46 (8) (2005) 083504, 6 pp.

[5] T. Cazenave, Semilinear Schrödinger Equations, Courant Lect. Notes Math., vol. 10, New York University, Courant Institute of Mathematical Sciences/American Mathematical Society, New York, Providence, RI, 2003.

[6] H. Gao, C. Bu, Neumann inhomogeneous boundary value problem for the $n+1$ complex Ginzburg-Landau equation, Appl. Math. Comput. 188 (1) (2007) 394-398.

[7] H. Gao, X. Gu, C. Bu, A Dirichlet inhomogeneous boundary value problem for a generalized Ginzburg-Landau equation, J. Math. Anal. Appl. 330 (1) (2007) 699-714.

[8] J. Holmer, The initial-boundary-value problem for the 1D nonlinear Schrödinger equation on the half-line, Differential Integral Equations 18 (6) (2005) 647-668.

[9] E.I. Kaikina, Forced cubic Schrödinger equation with Robin boundary data: large-time asymptotics, Proc. R. Soc. Lond. Ser. A Math. Phys. Eng. Sci. 469 (2159) (2013) 20130341, 16 pp.

[10] E.I. Kaikina, Asymptotics for inhomogeneous Dirichlet initial-boundary value problem for the nonlinear Schrödinger equation, J. Math. Phys. 54 (11) (2013) 111504, 15 pp.

[11] E.I. Kaikina, Inhomogeneous Neumann initial-boundary value problem for the nonlinear Schrödinger equation, J. Differential Equations 255 (10) (2013) 3338-3356.

[12] O. Kavian, A remark on the blowing-up of solutions to the Cauchy problem for nonlinear Schrödinger equations, Trans. Amer. Math. Soc. 299 (1) (1987) 193-203.

[13] I. Lasiecka, J.-L. Lions, R. Triggiani, Nonhomogeneous boundary value problems for second order hyperbolic operators, J. Math. Pures Appl. (9) 65 (2) (1986) 149-192.

[14] I. Lasiecka, R. Triggiani, Control Theory for Partial Differential Equations: Continuous and Approximation Theories. II. Abstract Hyperbolic-Like Systems over a Finite Time Horizon, Encyclopedia Math. Appl., vol. 75, Cambridge University Press, Cambridge, 2000.

[15] L. Ma, J. Wang, Inhomogeneous problem for the Ginzburg-Landau equation on two dimensional compact manifolds, Dyn. Partial Differ. Equ. 7 (2) (2010) 175-185.

[16] T. Özsarı, V.K. Kalantarov, I. Lasiecka, Uniform decay rates for the energy of weakly damped defocusing semilinear Schrödinger equations with inhomogeneous Dirichlet boundary control, J. Differential Equations 251 (7) (2011) $1841-1863$.

[17] T. Özsarı, Weakly-damped focusing nonlinear Schrödinger equations with Dirichlet control, J. Math. Anal. Appl. 389 (1) (2012) 84-97. 
[18] T. Özsarı, Global existence and open loop exponential stabilization of weak solutions for nonlinear Schrödinger equations with localized external Neumann manipulation, Nonlinear Anal. 80 (2013) 179-193.

[19] W. Strauss, C. Bu, An inhomogeneous boundary value problem for nonlinear Schrödinger equations, J. Differential Equations 173 (1) (2001) 79-91.

[20] R. Weder, The forced non-linear Schrödinger equation with a potential on the half-line, Math. Methods Appl. Sci. 28 (10) (2005) 1237-1255. 